\title{
The definition of cross polarization
}

\section{Ludwig, Arthur}

\section{Published in:}

I E E E Transactions on Antennas and Propagation

Publication date:

1973

\section{Document Version}

Publisher's PDF, also known as Version of record

Link back to DTU Orbit

\section{Citation (APA):}

Ludwig, A. (1973). The definition of cross polarization. I E E E Transactions on Antennas and Propagation, 21(1), 116-119.

\section{General rights}

Copyright and moral rights for the publications made accessible in the public portal are retained by the authors and/or other copyright owners and it is a condition of accessing publications that users recognise and abide by the legal requirements associated with these rights.

- Users may download and print one copy of any publication from the public portal for the purpose of private study or research.

- You may not further distribute the material or use it for any profit-making activity or commercial gain

- You may freely distribute the URL identifying the publication in the public portal

If you believe that this document breaches copyright please contact us providing details, and we will remove access to the work immediately and investigate your claim 
TABLE I

Comparison of Calculated $E_{z}$ For Frill with $a=0.003 \lambda$ and $b=0.005 \lambda$ Along $4 \tilde{j}^{\circ}$ Line

\begin{tabular}{|c|c|c|}
\hline$z=\rho($ in $\lambda)$ & Improved Form (14) & Numerical Differentiation [1, eq. (12)] \\
\hline $\begin{array}{l}0.0005 \\
0.0015 \\
0.0025 \\
0.0035 \\
0.0045 \\
0.0055 \\
0.0065 \\
0.0075 \\
0.0085 \\
0.0095\end{array}$ & $\begin{array}{l}0.2047159 E 02-j 0.1029276 E-03 \\
0.1688536 E 02-j 0.1029251 E-03 \\
0.9610034 E 01-j 0.1030192 E-03 \\
0.4387174 E 01-j 0.1031420 E-03 \\
0.2015952 E 01-j 0.1032633 E-03 \\
0.1019608 E 01-j 0.1032623 E-03 \\
0.5716718 E 00-j 0.1029731 E-03 \\
0.3493902 E 00-j 0.1029350 E-03 \\
0.2285606 E 00-j 0.1027498 E-03 \\
0.1576996 E 00-j 0.1028112 E-03\end{array}$ & $\begin{array}{l}0.2046579 E 02-j 0.1119673 E-03 \\
0.1688470 E 02-j 0.1044982 E-03 \\
0.9614460 E 01-j 0.1049856 E-03 \\
0.4387884 E 01-j 0.1037690 E-03 \\
0.2015988 E 01-j 0.1039268 E-03 \\
0.1019675 E 01-j 0.1042650 E-03 \\
0.5715361 E 00-j 0.1031028 E-03 \\
0.3491055 E 00-j 0.1038956 E-03 \\
0.2286559 E 00-j 0.1037456 E-03 \\
0.1576202 E 00-j 0.1035746 E-03\end{array}$ \\
\hline
\end{tabular}

Substitution of (8) and (9) into (7) and replacement of $\partial R / \partial \phi^{\prime}$ and $\partial R / \partial \rho$ by their respective equivalents,

$$
\frac{\partial R}{\partial \phi^{\prime}}=-\frac{\rho \rho^{\prime}}{R} \sin \left(\phi-\phi^{\prime}\right)
$$

and

$$
\frac{\partial R}{\partial p}=\frac{\rho-\rho^{\prime} \cos \left(\phi-\phi^{\prime}\right)}{R}
$$

reduce $E_{\varepsilon}$ to

$$
\begin{aligned}
E_{z}=-\frac{1}{4 \pi \ln (b / a)} \int_{\rho^{\prime}=a}^{b} \int_{\phi^{\prime}=0}^{2=} \frac{\partial}{\partial R}\left[\frac{\exp (-j k R)}{R}\right] \\
\cdot\left\{\frac{\rho^{\prime}-\rho \cos \left(\phi-\phi^{\prime}\right)}{R}\right\} d \phi^{\prime} d \rho^{\prime} .
\end{aligned}
$$

In (12), the term in the braces is recognized to be $\partial R / \partial \rho$; hence, $E_{z}$ simplifies to

$$
E_{z}=\frac{-1}{4 \pi \ln (b / a)} \int_{\rho^{\prime}=a}^{b} \int_{\phi^{\prime}=0}^{2 \pi} \frac{\partial}{\partial R}\left[\frac{\exp (-j k R)}{R}\right] \frac{\partial R}{\partial \rho^{\prime}} d \rho^{\prime} d \phi^{\prime}
$$

whose integrand is an exact differential with respect to $\rho^{\prime}$ and which becomes

$$
E_{z}=\frac{-1}{4 \pi \ln (b / a)} \int_{\phi^{\prime}=0}^{2 \pi}\left[\frac{\exp (-j k R)}{R}\right]_{\rho^{\prime=a}}^{b} d \phi^{\prime} .
$$

Equation (14) is exact and applies for all observation points not on the frill proper, but unlike [1, eq. (12) ], no numerical differentiation is required. This results in increased efficiency and accuracy since only a simple numerical integration is now needed. Equation (14) reduces readily to the axial form $(\lim \rho \rightarrow 0$ ) given earlier in [1, eq. (25) $]$. A comparison of the calculated $E_{z}$ using both (14) and the numerical differentiation form of $[1$, eq. (12) $]$ is of interest and is presented in Table $I$. Fields are observed along a $45^{\circ}$ line $(\rho=z)$ for increasing $\rho$ where one notices that the agreement is quite close. Equation (14) is found to be much more effcient, its evaluation requiring approximately one-tenth the computer time needed for [1, eq. (12)].

The simplification thus achieved for the computation of $E_{z}$ is perhaps somewhat fortuitous in that the same procedure does not reduce the complexity for calculating $E_{\rho}$. Thus the numerical differentiation process given in [1] is still needed for the computation of $E_{\rho}$. However, the simpler form for $E_{z}$ in (14) can certainly be employed to good advantage in a study of ground-plane mounted antennas, particularly, in an analysis of arrays of parallel monopoles such as the Yagi-Uda and log-periodic antennas, where the only knowledge needed of the excitation from the primary frill drive is the $z$ component of electric field $E_{z}$ on the wire elements.

\section{ACKNOW LEDGMENT}

The authors acknowledge a reviewer's suggestion which rendered the above development more direct. Also, they wish to mention, as was pointed out to them by Prof. B. K. Park, that (14) can be obtained in a formal manner by employing several vector identifies, symmetry properties of $R$, and Stokes' theorem.

\section{REFERENCES}

[1] L. L. Tsai, "A numerical solution for the near and far flelds of an annular ring of magnetic current", $I E E E$ Trans. Antennos Propagat. vol. AP-20, pp. 569-576, Sept. 1972 .

\section{The Definition of Cross Polarization}

\section{ARTHUR C. LUDWIG}

Abstract-There are at least three different definitions of cross polarization used in the literature. The alternative definitions are discussed with respect to several applications, and the definition which corresponds to one standard measurement practice is proposed as the best choice.

The use of orthogonal polarization to provide two communications channels for each frequency band has led to interest in the polarization purity antennatterns. It is a surprising fact that there is no universally accepted definition of "cross polarization" at the present, and at least three different definitions have been used either explicitly or implicitly in the literature. The IEEE Standard [1] definition is "The polarization orthogonal to a reference polarization." For circular polarization this is adequate, but for linear or elliptical polarization the direction of the reference polarization must still be defined.

We will first briefly present the definitions known to the author. Only the case of nominally linear polarization will be considered since the extension to elliptical polarization is straightforward. The three alternative definitions are: 1) in a rectangular coordinate system, one unit vector is taken as the direction of the reference polarization, and another as the direction of eross polarization [2]; 2) in a spherical coordinate system the same thing is done using the unit vectors tangent to a spherical surface [3], [4]; and 3) reference and cross polarization are defined to be what one measures when antenna patterns are taken in the usual manner [2, pp.

Manuscript received May 30, 1972; revised August 3,1972 . This work was supported by the European Space Research and Technology Centre.

The author was with the Laboratory of Electromagnetic Theory, Technical University of Denmark. He is now with the Jet Propulsion Laboratory, California Institute of Technology, Pasadena, Calif. 91103. 
557-564], [5]. These cases, which will be defined more precisely, are illustrated in Fig. 1.

Two different cases in which cross polarization is of interest may be distinguished: 1) deseribing the secondary radiation pattern of a complete antenna system, and 2) describing the source or primary field distribution. In the first case, it is desirable to have a definition which applies for all pattern angles, and which is easily related to channel interference, or other requirements such as ensuring that cross polarized fields do not exceed angiven sidelobe specification. In the second case, it is desirable to have a simple relationship between source cross polarization and secondary pattern polarization. A second common application is the calculation of antenna feed or aperture illumination efficiency, where cross polarization must be included as a gain loss factor [6]. In this case, "cross polarization" really means fields which are antisymmetric in the aperture and therefore do not contribute to radiation on-axis. So it is desirable that the definition be consistent with this usage also.

We will now express the three definitions precisely and in terms of the same antenna pattern coordinate system as illustrated in Fig. 1. This will be done by deriving unit vectors $\hat{i}_{\text {ref }}$ and $\hat{i}_{\text {oross }}$ such that

$$
\begin{gathered}
E \cdot \hat{i}_{\mathrm{ref}} \equiv \text { the reference polarization component of } E \\
E \cdot \hat{i}_{\text {cross }} \equiv \text { the cross polarization component of } E .
\end{gathered}
$$

Definition 1 is a trivial case with

$$
\begin{gathered}
\hat{i}_{\text {ref }}^{(1)}=\hat{i}_{y}=\sin \theta \sin \phi \hat{i}_{r}+\cos \theta \sin \phi \hat{i}_{\theta}+\cos \phi \hat{i}_{\phi} \\
\hat{i}_{\text {eross }}{ }^{(1)} \equiv \hat{i}_{x}=\sin \theta \cos \phi \hat{i}_{\tau}+\cos \theta \cos \phi \hat{i}_{\theta}-\sin \phi \hat{i}_{\phi} .
\end{gathered}
$$

For the second case, the polarization unit vectors are defined in a system of rectangular and spherical coordinates related to the system shown in Fig. 1 by

$$
\tilde{x}=x \quad \tilde{y}=z \quad \tilde{z}=-y .
$$

Then, by definition 2 , we have

$$
\begin{aligned}
\hat{i}_{\text {ref }}^{(2)} & \equiv \hat{i}_{\tilde{\theta}} \\
& =\frac{-\sin ^{2} \theta \sin \phi \cos \phi \hat{i}_{x}+\left(1-\sin ^{2} \theta \sin ^{2} \phi\right) \hat{i}_{y}-\sin \theta \cos \theta \sin \phi \hat{i}_{z}}{\left\{1-\sin ^{2} \theta \sin ^{2} \phi\right\}^{1 / 2}}
\end{aligned}
$$$$
=\frac{\sin \phi \cos \theta \hat{i_{\theta}}+\cos \phi \hat{i}_{\phi}}{\left\{1-\sin ^{2} \theta \sin ^{2} \phi\right\}^{1 / 2}}
$$

$$
\begin{aligned}
\hat{i}_{\text {oross }}^{(2)} & \equiv-\hat{i}_{\phi}^{-} \\
& =\frac{\cos \theta \hat{i_{x}}-\sin \theta \cos \phi \hat{i}_{z}}{\left\{1-\sin ^{2} \theta \sin ^{2} \phi\right\}^{1 / 2}} \\
& =\frac{\cos \phi \hat{i}_{\theta}-\cos \theta \sin \phi \hat{i}_{\phi}}{\left\{1-\sin ^{2} \theta \sin ^{2} \phi\right\}^{1 / 2}} .
\end{aligned}
$$

It should be noted that these equations depend on the choice of the relative orientation of the pattern and polarization coordinate systems. There are really two cases of definition 2 obtained by interchanging the subscripts ref and cross in (4). If this interchange is made in definitions 1 or 3 , one obtains a result equivalent to rotating the coordinate system by $90^{\circ}$ about the $z$ axis (neglecting unimportant sign changes), but this is not true for definition 2. It is straightforward to show that

$$
\begin{aligned}
& \hat{i}_{\mathrm{ref}}^{(2)}(\theta, \phi) \cdot \hat{i}_{\mathrm{ref}}^{(2)}\left(\theta, \phi+90^{\circ}\right) \\
& =\hat{i}_{\mathrm{eross}}{ }^{(2)}(\theta, \phi) \cdot \hat{i}_{\mathrm{erogs}}{ }^{(2)}\left(\theta, \phi+90^{\circ}\right) \\
& =\frac{\sin ^{2} \theta \sin \phi \cos \phi}{\left\{\left(1-\sin ^{2} \theta \sin ^{2} \phi\right)\left(1-\sin ^{2} \theta \cos ^{2} \phi\right)\right\}^{1 / 2}} .
\end{aligned}
$$

TOP: DIRECTION OF THE REFERENCE POLARIZATION BOTTOM: DIRECTION OF THE CROSS POLARIZATION

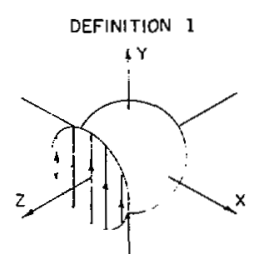

4Y

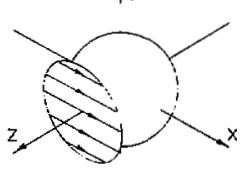

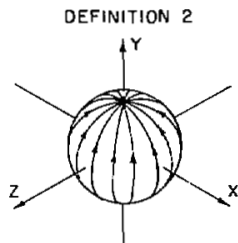

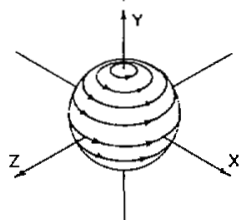

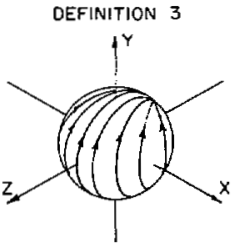

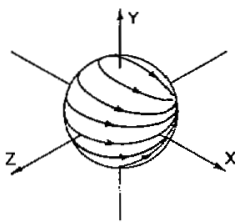

Fig. 1. Alternate polarization definition.

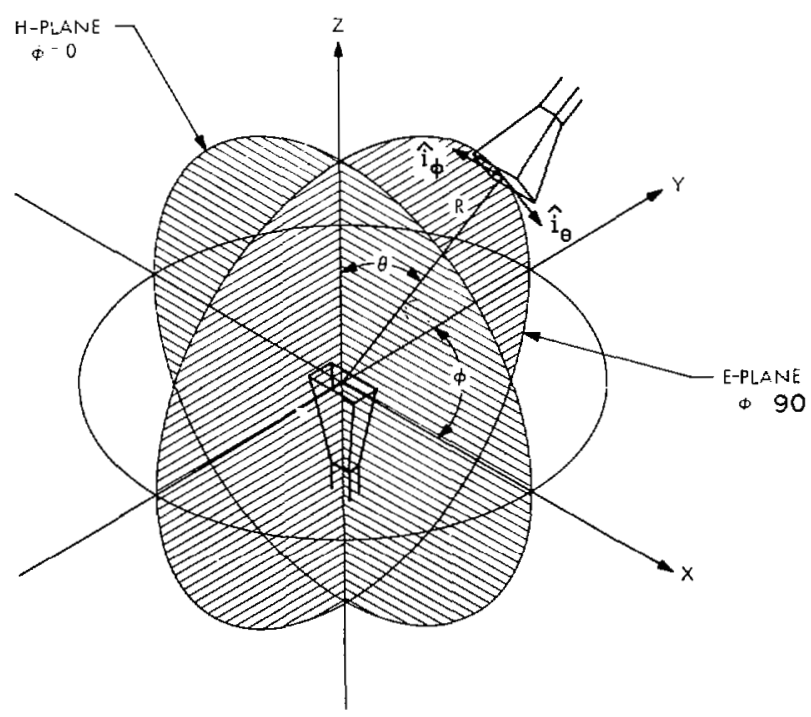

Fig. 2. Antenna pattern measurement system.

$$
\text { -. }
$$

The third definition is simple in practice, but tricky to formulate precisely. The pattern measurement method that will be described is probably the one most commonly used by antenna engineers. It is sometimes presented as a standard/method for testing feeds or small aperture antennas, [2, pp. 557 564$]$, [7], but it can also be used for large antennas. In the terminology used by ScientificAtlanta, this method may be implemented by mounting the antenna being tested, shown at the origin of the system as illustrated in Fig. 2, on a model tower or an elevation-over-azimuth positioner with an auxiliary polarization axis [8]. The elevation angle is always zero ( $z$ axis horizontal) so this axis is actually not required. Each pattern cut begins at $\theta=0$, where the polarization axis is used to set the pattern cut angle $\phi$ by rotating the antenna being tested about the $z$ axis. The probe is rotated about its axis by a second polarization positioner to align the probe polarization parallel to the polarization of the antenna being tested for a principal polarization pattern, or orthogonal to the polarization of the antenna being tested for a cross polarization pattern. Note that the orientation of the polarization of the antenna being tested, at the point $\theta=0$, is the basic polarization reference direction by definition. Then a pattern is taken by varying $\theta$ by rotating in azimuth. This is equivalent to the probe traversing a great circle as illustrated in Fig. 2. The probe remains fixed about its axis so it retains the same relative orientation with respect to the unit vectors $i_{\theta}$ and $\hat{i}_{\phi}$. Therefore, the measured pattern is given by

$$
M(\theta)=E(\theta, \phi) \cdot\left\{\sin \beta i_{\theta}+\cos \beta \hat{i_{\phi}}\right\}
$$


TABLE I

Source Current Contributions to Radiated Pattern Cross Polarization

\begin{tabular}{|c|c|c|c|c|}
\hline & \multirow{2}{*}{$\begin{array}{c}\text { Source } \\
\text { Current } \\
\text { Polarization }\end{array}$} & \multicolumn{3}{|c|}{ Contribution to Secondary Pattern Cross Polarization } \\
\hline & & Definition 1 & Definition 2 & Definition 3 \\
\hline \multirow{3}{*}{$\ddots$} & $\hat{i}_{z}$ & $1-\sin ^{2} \theta \cos ^{2} \phi$ & $\frac{\cos \theta}{\left(1-\sin ^{2} \theta \sin ^{2} \phi\right)^{1 / 2}}$ & $1-\cos ^{2} \phi(1-\cos \theta)$ \\
\hline & $\hat{\imath}_{y}$ & $-\sin ^{2} \theta \sin \phi \cos \phi$ & 0 & $-(1-\cos \theta) \sin \phi \cos \phi$ \\
\hline & $\hat{v}_{z}$ & $-\sin \theta \cos \theta \cos \phi$ & $\frac{-\sin \theta \cos \phi}{\left(1-\sin ^{2} \theta \sin ^{2} \phi\right)^{1 / 2}}$ & $-\sin \theta \cos \phi$ \\
\hline
\end{tabular}

where $E(\theta, \phi)$ is the field of the transmitting antenna, and the pattern cut angle $\phi$ and probe polarization angle $\beta$ are fixed for a given pattern. For a transmitted field polarized in the $\hat{i}_{y}$ direction at $\theta=0$, this alignment procedure leads to $\beta=\phi$ for a reference polarization pattern $R(\theta, \phi)$ and $\beta=\phi \pm 90^{\circ}$ for a cross polarized pattern $C(\theta, \phi)$. Therefore, ignoring unimportant sign differences,

$$
\begin{aligned}
& R(\theta, \phi)=E(\theta, \phi) \cdot\left\{\sin \phi \hat{i}_{\theta}+\cos \phi \hat{i}_{\phi}\right\} \\
& C(\theta, \phi)=E(\theta, \phi) \cdot\left\{\cos \phi \hat{i}_{\theta}-\sin \phi \hat{i}_{\phi}\right\} .
\end{aligned}
$$

This is equivalent to

$$
\begin{aligned}
\hat{i}_{\mathrm{ref}}^{(3)} \equiv & \sin \phi \hat{i}_{\theta}+\cos \phi \hat{i}_{\phi} \\
= & -(1-\cos \theta) \sin \phi \cos \phi \hat{i}_{x} \\
& +\left\{1-\sin ^{2} \phi(1-\cos \theta)\right\} \hat{i}_{y}-\sin \theta \sin \phi \hat{i}_{z} \\
\hat{i}_{\mathrm{orosg}}^{(3)=} & \cos \phi \hat{i}_{\theta}-\sin \phi \hat{i}_{\phi} \\
= & \left\{1-\cos ^{2} \phi(1-\cos \theta)\right\} \hat{i}_{x} \\
& -(1-\cos \theta) \sin \phi \cos \phi \hat{i}_{y}-\sin \theta \cos \phi \hat{i}_{z} .
\end{aligned}
$$

The procedure defined in the preceding makes it easy to avoid probe polarization misalignment which is a severe pitfall in cross polarization measurements. To show this, suppose that $\beta=\left(\phi+90^{\circ}\right)+\epsilon$. Then from (6) and (7)

$$
M(\theta)=C(\theta, \phi) \cos \epsilon-R(\theta, \phi) \sin \epsilon .
$$

To illustrate what this means in practice, suppose that the true cross polarization is negligible, but a misalignment of $\epsilon=1.5^{\circ}$ is present. Then one would measure a "cross polarized" pattern which is actually the reference polarization pattern suppressed by $31.6 \mathrm{~dB}$ (i.e., $\sin 1.5^{\circ}$ ). Cross polarization patterns which do not have a null on axis are a symptom of this error.

Before discussing the relative merits of these definitions, it is necessary to relate the polarization of source currents $J$ to the polarization of the radiated pattern $E(\theta, \phi)$, which is given by [9]

$$
E(\theta, \phi)=F(\theta, \phi)-\left\{\boldsymbol{F}(\theta, \phi) \cdot \hat{i}_{\tau}\right\} \hat{i}_{r}
$$

where

$$
F(\theta, \phi)=-\frac{j \omega \mu}{4 \pi} \frac{\exp (-j k r)}{r} \int J \exp \left(-j k \omega \cdot \hat{i}_{r}\right) d S
$$

and the undefined terms are unimportant for present purposes. In order to directly relate the components of $J$ at any point to the components of $F$ at any pattern angle, it is necessary to use unit vectors which do not vary as a function of either the pattern coordinates or the integration coordinates; the only apparent choice is rectangular unit vectors. Then the $x, y$, and $z$ components of $J$ are uniquely related to the $x, y$, and $z$ components of $F$, respectively. However, the components of $F$ and $E$ do not have such a simple relation. From (10a) it can be found that they are coupled by a polarization matrix.

$$
\left[\begin{array}{c}
E_{x} \\
E_{y} \\
E_{z}
\end{array}\right]=\left[\begin{array}{ccc}
1-\sin ^{2} \theta \cos ^{2} \phi & -\sin ^{2} \theta \sin \phi \cos \phi & -\sin \theta \cos \theta \cos \phi \\
-\sin ^{2} \theta \sin \phi \cos \phi & 1-\sin ^{2} \theta \sin ^{2} \phi & -\sin \theta \cos \theta \sin \phi \\
-\sin \theta \cos \theta \cos \phi & -\sin \theta \cos \theta \sin \phi & 1-\cos ^{2} \theta
\end{array}\right]
$$

Similar factors relating the source current polarization to the radiated pattern cross polarization, for all three definitions, are given in Table I. It is seen that in all cases the dominant cause of cross polarization is the $i_{x}$ source currents. The $\hat{i}_{y}$ source current contribution to cross polarization is suppressed by a factor which is in excess of $52 \mathrm{~dB}$ for $\theta<4^{\circ}$. The $\hat{i}_{z}$ contribution is suppressed by a factor which is in excess of $23 \mathrm{~dB}$ for $\theta<4^{\circ}$. Therefore, it is reasonable to define the $\hat{i}_{x}$ component as the cross polarized source currents, and to use the common terminology of longitudinal currents for the $\hat{i}_{2}$ component to distinguish the fact that it is a far less serious source of secondary pattern cross polarization. Of course, the $\hat{i}_{y}$ currents are the reference polarization currents.

Now we will compare the three definitions. Since the far-field fields of any antenna are tangent to a spherical surface, it is immediately apparent that definition 1 is fundamentally inappropriate for these applications. However, as noted above, it is the only apparent definition which applies to the case of source currents. Definitions 2 and 3 involve unit vectors tangent to a sphere so they are appropriate for the case of primary or secondary fields. For evaluating secondary patterns for the application of orthogonal channels, we postulate the following ideal case: the transmitting antenna has two ports, which radiate two patterns that are orthogonal at every pattern angle in the coverage region. Clearly, it is then possible to receive the two channels without any interference anywhere in the coverage region (in fact, this is far easier than the transmit problem since the receiving antenna must be free of cross polarization only very close to its axis). Now, since any field is everywhere orthogonal to some other field, this still leaves the "perfect" pattern undefined. A logical choice is a pattern which is orthogonal to itself after a $90^{\circ}$ rotation about the $z$ axis. This is also a realistic choice since it corresponds to adding an orthomode transducer to an otherwise circularly symmetric antenna. A pattern with no cross polarization by definitions 1 or 3 satisfy this requirement; this is not true for definition 2 as shown by (5), and as previously pointed out by Kreutel and Di Fonzo [4].

For relating source current distributions to secondary patterns, it is logical that a perfect source distribution radiate a perfect pattern; only definition 2 is compatible with this requirement, as shown in Table I.

For evaluating primary feed patterns for paraboloidal reflectors a logical requirement is that a perfect feed cause a perfect surface 
current distribution. By definition 2 an infinitesmial electric dipole pattern contains no cross polarization (if the subscripts ref and cross are reversed in definition 2 , an infinitesimal magnetic dipole is perfect). However, it is well known that this feed causes substantial cross polarization [10]. This question has also been treated in an excellent paper by Koffman [11], where it is shown that a necessary and sufficient condition for zero cross polarized surface currents is

$$
E_{\theta} \cos \phi=E_{\phi} \sin \phi
$$

From (8) it can be seen that this is identically equivalent to definition 3. Koffman gives an example of a Huygens source as satisfying (12). It is also possible to show that a physically circular feed with equal $E$ - and $H$-plane amplitude and phase patterns is also perfect by this definition [6]. From (2) it may be seen that a perfect feed by definition 1 does not satisfy (12) either, so only definition 3 satisfies this requirement.

Finally, it has been previously shown by the author [6] that a feed with no cross polarization by definition 3 is optimum from the viewpoint of antenna aperture efficiency. Again, this is not true for either definition 1 or 2 .

From the preceding discussion, it is clear that definition 1 is the proper choice for describing source current polarizations. It is the author's opinion that definition 3 is the best choice for describing antenna patterns. The only disadvantage of definition 3 is its imperfect relationship with the source current definition. However, this point is muddled in any case by the existence of longitudinal source currents. Definition 2 has the disadvantage of two perfect secondary patterns rotated $90^{\circ}$ with respect to each other not being orthogonal, the serious point that a perfect primary pattern can produce a very poor secondary pattern, and its incompatibility with feed efficiency usage.

As an illustration of how definition 2 can be misleading, it may be noted that the cross polarized currents on a paraboloid illuminated by an infinitesimal electric dipole are frequently attributed to the reflector curvature. As a result, it is widely accepted that increasing the reflector $f / D$ ratio substantially reduces cross polarization [12]. By definition 3, an electric dipole has substantial cross polarization which increases rapidly with increasing pattern angle (i.e., as the $E$ - and $H$-plane edge illuminations diverge). A paraboloid with lower $f / D$ subtends a larger pattern angle, and this is the reason that the cross polarization in the secondary pattern becomes worse. If the $E$ - and $H$-plane edge illumination is held constant it is not difficult to show that the cross polarized currents are actually independent of the $f / D$ ratio. Also, for certain practical feeds it has been pointed out that cross polarization may actually increase with increasing $f / D$ [4]. The $f / D$ ratio does effect the longitudinal currents, and also can have an effect in the case of defocused feeds, but with a proper definition it is seen that secondary pattern cross polarization is far less dependent on the $f / D$ ratio than it seems from definition 2 .

\section{ACKNOWLEDGMENT}

The author would like to acknowledge several interesting discussions on this topic with D. C. Patel of the European Space Research and Technology Centre.

\section{REFERENCES}

[1] "IEEE standard definitions of terms for antennas," IEEE Trans. 11] Antennas Propagat., vol. AP-17, Dp. 262-269, May 1969. [2] Antennas Sropagat. Vol. AP-17, pp. 262-269, May 1969. New York:

[3] V.P. Narbut and $\mathrm{X}$. S. S. Khmel'nitskaya, "Polarization structure of radiation from axisymmetric reflector antennas," Radio Eng.

[4] Dlectron. Phys., vol. 15, pp. 1786-1796, 1970 . antennas for frequency reuse," presented at G-AP Int. Symp. paper $12-1$, Sept. 1971 .

[5] J. D. Kraus, Antennas. New York: McGraw-Hill, 1950, ch. 15.

[6] A. C. Ludwig, "Antenna feed efficiency." Jet Prop. Lab., Calif. Inst. Technol., Pasadena, Space Programs Summary 37-26, vol. IF. pp. 200-208, 1965 .

[7] "Test procedures for antennas," IEEE Publ. Yo. 149, Jan. 1965.

81 J. S. Hollis, T, J. Lyon, and L. Clayton, "Microwave antenna measurements," Scientific-Atlanta, Inc., Atlanta, Ga., Tech. Rep., 1970.
[9] W. V. T. Rusch and P. D. Potter, Analysis of Reflector Antennas. New York: Academic Press, 1970, p. 44.

[10] E. M. T. Jones, "Paraboloid reflector and hyperboloid lens antennas," IRE Trans. Antennas Propagat., vol. AP-2, pp. 119-127, July 1954.

[11] I. Koffman, "Feed polarization for parallel currents in reflectors generated by conic sections," IEEE Trans. Antennas Propagat., Yol. AP-14, pp. 37-40, Jan. 1966 .

[12] R. E. Collin and F. J. Zucker, Anienna Theory. New York: McGraw-Hill, 1969, part 2, p. 44 .

\section{see AP Nov. 73 p 917}

\section{Distortion of Electromagnetic Pulses Undergoing Total Internal Reflection from a Moving Dielectric Half-Space}

\section{RATTAN, A. K. CHAKRAVARTI, AND G. D. GAUTAMA}

Abstract-Using the covariance of Maxwell's equations and the phase invariance principle of plane waves, distortion of the electromagnetic pulses undergoing total internal reflection from a moving dielectric half-space has been studied. It is concluded that the distortion of the reflected pulse depends strongly upon the velocity, direction of motion, and angle of incidence.

In recent years, much concern has been shown to the reflection and transmission of electromagnetic waves by moving media because of its direct relevance to the problems of current interest, viz., satellite communications and reentry vehicles etc. With the plane monochromatic wave propagation problem practically resolved, it is worthwhile to investigate the more practical problem of reflection of pulses from these media. In this communication, the distortion of electromagnetic pulses undergoing total internal reflection from a moving dielectric half-space is studied.

Since an electromagnetic pulse can be thought of as the superposition of plane electromagnetic waves, Yeh's [1] treatment for moving dielectric half-space for studying reflection of electromagnetic waves can be carefully extended to this case, using Fourier analysis technique.

Let the pulse be incident from the denser medium side $z<0$ to the rarer mediuin side $z>0$. If $E_{i}(t)$ represents the incident pulse which is impinging at an angle $\theta_{0}>\sin ^{-1}\left(\epsilon_{3} / \epsilon_{1}\right)$ at the boundary $z=0$, where $\epsilon_{2}$ and $\epsilon_{1}$, are the permittivities of the rarer and denser media, respectively, we have

$$
E_{i}\left(t_{i}\right)=\frac{1}{2} \int_{0}^{\infty} g(\omega) \exp \left(i \omega t_{i}\right) d \omega+\frac{1}{2} \int_{0}^{\infty} g^{*}(\omega) \exp \left(-i \omega t_{i}\right) d \omega
$$

where $g^{*}(\omega)$ is complex conjugate of $g(\omega)$

$$
\begin{aligned}
& t_{i}=\frac{1}{\omega}\left(k_{z y} x+k_{z} z-\omega t\right) \\
& k_{x}=k_{0} \sin \theta_{0} \\
& k_{z}=-k_{0} \cos \theta_{0} \\
& k_{0}=\omega\left(\mu_{0} \epsilon_{1}\right)^{1 / 2}
\end{aligned}
$$

and $\theta_{0}$ is also the angle between propagation vector $k$ and positive $z$ axis in $x z$ plane.

The amplitude of the spectral component $g(\omega)$ is given by inverse Fourier transform of $E_{i}\left(t_{i}\right)$ as

$$
g(\omega)=\frac{1}{\pi} \int_{-\infty}^{+\infty} E_{i}\left(t_{i}\right) \exp \left(-i \omega t_{i}\right) d t_{i}
$$

Manuscript received May 31, 1972. This work was supported by the U.S. National Oceanographic and Atmospheric Administration and the Indian CSIR.

The authors are with the Department of Physies, Indian Institute of Technology, New Delhi-29, India. 